\title{
Design Overview of a Toroidal Fast-Field Cycling electromagnet
}

\author{
João T. Cunha ${ }^{1}$, Pedro J. Sebastião ${ }^{1}$, António Roque ${ }^{2,3}$, Vitor Vaz da Silva ${ }^{4,5}$ and Duarte M. Sousa ${ }^{6}$ \\ ${ }^{1}$ CeFEMA, Instituto Superior Técnico, Lisboa, Portugal \\ Av. Rovisco Pais 1, 1049-001 lisboa, Portugal \\ +351 21841 9210, joaotiagocunha@tecnico.ulisboa.pt, pedro.jose.sebastiao@tecnico.ulisboa.pt \\ ${ }^{2}$ Department of Electrical Engineering \\ ESTSetúbal/Instituto Politécnico de Setúbal, Campus of IPS, Estefanilha, 2914-761 Setúbal, Portugal, Phone/Fax: +351 \\ 265790000; antonio.roque@estsetubal.ips.pt \\ ${ }^{3}$ INESC-ID, \\ Av. Alves Redol 9, 1000-029 Lisboa, Portugal, Phone/Fax: +351 213100300/ +351 213100235 Lisboa, Portugal
}

\author{
${ }^{4}$ ADEETC - ISEL, Instituto Politécnico de Lisboa, Lisboa, Portugal \\ vsilva@deetc.isel.ipl.pt \\ ${ }^{5}$ CTS, Universidade Nova de Lisboa, Caparica, Portugal \\ ${ }^{6}$ DEEC, AC Eergia, Insituto Superior Técnico, Universidade de Lisboa \\ Av. Rovisco Pais 1, 1049-001 lisboa, Portugal \\ +35121841 7429, duarte.sousa@ tecnico.ulisboa.pt
}

\begin{abstract}
In this paper, the design and development of a novel Fast-Field Cycling (FFC) Nuclear Magnetic Resonance (NMR) relaxometer's electromagnet is described. This magnet is tailored to increase the relaxometers's usability, by increasing its portability capacities. It presents a compact toroidal shaped iron core, allowing to operate in a field range of 0 to $0.21 \mathrm{~T}$, with high field homogeneity (less than $800 \mathrm{ppm}$ in a volume of $\approx 0.57 \mathrm{~cm}^{3}$ ), low power consumption and reduced losses (about $40 \mathrm{~W}$ ). The simulation software COMSOL Multiphysics ${ }^{\circledR}$ is used to characterize the induced magnetic field, the heating and the cooling effects. The proposed optimized layout constitutes an innovative solution for FFC magnets.
\end{abstract}

Key words. Fast-Field Cycling, Nuclear Magnetic Resonance, NMR electromagnet, Portable NMR.

\section{Introduction}

The Fast-Field Cycling (FFC) Nuclear Magnetic Resonance NMR technique explored in this work is originated from the fact that using the more conventional relaxometry techniques (such as inversion-recovery) it is very impractical, or even impossible, to perform studies at lower magnetic fields [1]. This is due, mainly, to the decrease of the signal-to-noise ratio at low fields. he FFC technique allows to overcome this weakening of the NMR induction signal at lower fields by placing the sample under the influence of a cycling magnetic field [2]. Doing so, it becomes possible to perform measurements at a low intensity field, but with the signal acquisition only being performed at a higher intensity field (and thus with a good signal-to-noise ratio) [3-4]. To accomplish the referred purposes, an optimized design of the FFC NMR eletromagnet is required [5-6].

This circumstance can be rather problematic since FFC experiments are very sensitive to the thermal stability of the magnets, which can affect the intensity of the magnetic fields and the switching times between field levels. In situations where the cooling system is not able to stabilize the temperature of the magnet, it becomes necessary to have certain periods during the relaxometry field cycles where the magnet power is reduced to very low values [7]. A possible solution to reduce power and cooling demands, and decrease operating costs, is to use ferromagnetic materials for the magnet's core. Such materials present magnetic permeabilities significantly higher than that of air, establishing therefore a medium where the magnetic flux can be magnified. As a result, electrical current (and, thus, cooling) demands are considerably reduced. This is precisely the approach followed in this work, with the design of an iron-cored magnet [8], which constitutes a step forward in the design of FFC magnets opening the possibility of having portable equipments. In addition, this novel layout can allow to perform FFC measurements with samples in situ instead of using samples in vitro. 


\section{Fast-Field Cycling and the Novel Magnet System}

A FFC experiment intends to properly observe the behaviour of the nuclear spin system, several cycles need to occur with varying experimental parameters [6]. Thus, after a cycle, a recycle delay follows, allowing for the reset of the polarization and thermal equilibrium, so that the next cycle can begin [9-11].

This technique allows for the magnetization to reach its asymptotic value via relaxation at a low intensity field, which can be set to any desired value, while the signal detection is still performed at a high intensity field [5], which is crucial in order to have a good signal-to-noise ratio. The main component of a FFC relaxometer is the magnet system [12-13]. As the ultimate purpose of this work was the development of a highly portable FFC relaxometer, a magnet with very particular characteristics was created. Its defining one is the capacity to freely rotate over all three axes. This allows to have easier access to samples of different size and shape by being able to adapt the relaxometer to them, instead of the opposite, thus reducing the level of interference with their natural state. To that end, we propose a toroidal magnet's core, with a gap on one of its sides for sample insertion. The coils are wound over this structure, being distributed symmetrically around it (see Fig. 1).

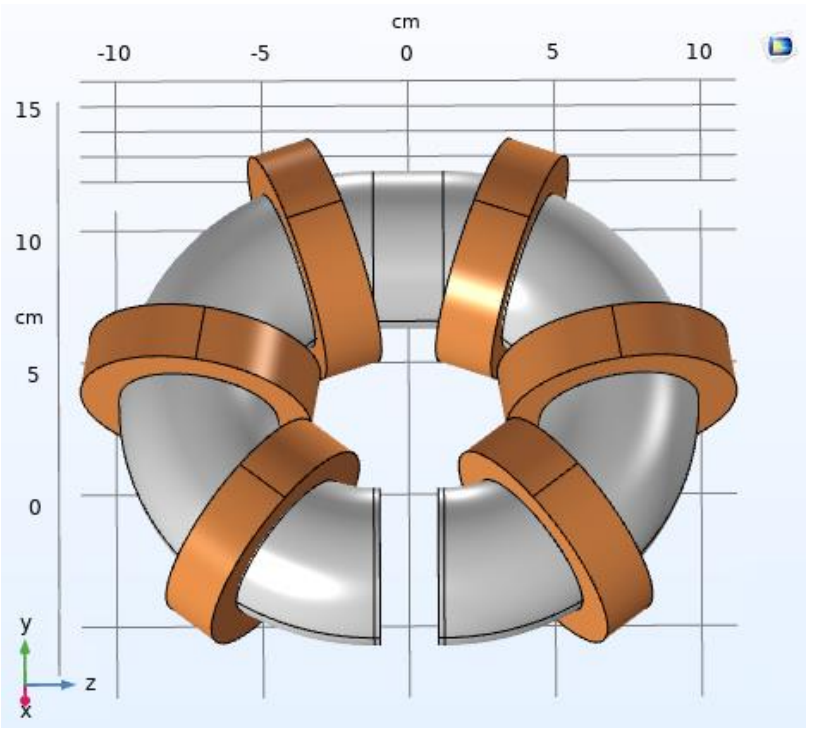

Fig. 1. Envisioned magnet system specially designed to increase the relaxometer's portability. The coils are represented in brown, and the core in grey.

The iron core presents a relatively high permeability (of the order of 1000, compared with just 1 for air). This means that the iron core provides a path of much less resistance than air for the magnetic field lines, supporting the development and magnification of the magnetic flux. The core is also a fundamental structural component of the magnet, as it serves as the physical support for the coils. In terms of its composition, instead of using a solid iron core, two different approaches to reduce core losses, were studied: use iron fillings to form the core, or create a laminated core using thin iron sheets.

To to obtain the magnet's parametric configuration, a series of simulations were performed using COMSOL
Multiphysics®. The objective of these simulations was to evaluate the impact that every controllable parameter had on the magnet's characteristics, thus allowing to establish an optimal equilibrium between them.

The main observations/results obtained for each of the analyzed parameters are summarized as follows:

- Iron core dimensions: The dimensions of the iron core greatly influence the characteristics of the induced magnetic field in the sample site, namely its intensity and homogeneity. Two parameters were evaluated: the radius of the toroidal iron core and its tube radius. On one hand, the greater both these dimensions were, the more intense and homogeneous was the magnetic field. On the other hand, greater dimensions meant larger size and weight of the magnet, which went against the objective of increasing its portability.

- Number of coils: It was rather straightforward to understand that the larger the number of coils used, the stronger would be the induced magnetic field, if everything else is kept constant. However, increasing the number of coils produces other limitations, namely: a decrease of available space and an increase of cooling. It was also concluded that, to reach the required field intensity of $\approx 0.21 \mathrm{~T}$ in the sample site, it would be preferable to use more coils with smaller dimensions (less turns per coil) than less coils with larger dimensions. Doing so, the homogeneity in the sample site increased, while the power demands and the inductance of the coils decreased.

- Number of turns per coil: The intensity of the induced magnetic field was directly related to the number of turns per coil. Nonetheless, as seen in the previous point, it was preferable to slightly decrease the number of turns per coil, while increasing the total number of coils. In fact, as the number of turns determines the height and width of the coils, more turns resulted in larger coils, which in turn caused the power demands (and Joule losses) to increase. - Coils position: The position of the coils relative to the sample gap was found to affect the homogeneity and intensity of the field in the sample site. For homogeneity reasons, a certain minimum distance had to be kept between the first coil in each side of the core and the gap. Nonetheless, if the coils were placed too far from the gap, the field intensity and homogeneity would also decrease, as more leakage would occur throughout the core. Also, some distance had to be kept between consecutive coils, to increase the cooling efficiency of the magnet system.

- Sample gap length: It was concluded that the smaller the gap was, the higher was the intensity and homogeneity of the magnetic field in the sample site. On the other hand, considering the objective of increasing the magnet's usability capacities, the gap should be as large as possible, so as to allow for bigger, more varied samples to be studied. It was also necessary to take into account that the gap length limits the width of the coils, since they are inserted (and removed) through the gap.

As the main objective was to increase the relaxometer's usability, the first priority was to keep the overall dimensions and weight of the magnet system as small as possible. In any case, the gap had to be sufficiently large to give us enough flexibility in terms of sample diversity. Additionally, the homogeneity and intensity of the induced 
magnetic field still had to fulfill minimum quality parameters, to ensure a satisfactory level of performance of the magnet.

Taking all this into consideration, the optimal magnet configuration obtained has the design parameters, presented in Table I.

Table I. - Type Sizes

\begin{tabular}{c|c} 
Gap length $[\mathrm{cm}]$ & 2 \\
\hline Torus radius $[\mathrm{cm}]$ & 6 \\
\hline Number of coils & 6 \\
\hline Turns per coil & 146 \\
\hline Coil width/height $[\mathrm{cm}]$ & $2 / 1.3$
\end{tabular}

\section{Magnetic Field, Fringing effect and Cooling}

A series of different combinations of magnet parameters that were tested in order to confirm that this was indeed a configuration close to optimal. These combinations of magnet characteristics included different field homogeneities at the sample site, different coil inductances, diffent core weight/volumen ratios, and different core power losses. Additionally, multiple plots describing the induced magnetic field were analyzed. An example of one of those plots is presented in Fig. 2, where a detailed view of the magnetic field inside the iron core is shown, along with a surface plot of the magnetic field in the middle of the sample gap.

As expected for a toroidal core geometry, this plot shows that the field is more intense on the inside of the torus, particularly in the zones where the coils were placed. Near the sample gap the magnetic field reaches its lower intensity, which is also expected being that this is the point furthest away from any coils. The well defined field map attests to the high homogeneity of the magnetic field in the sample site.

Fig. 2. Volume, slice and linear plots describing the magnetic field inside the core and in the sample gap of the magnet core filling system: a) Magnetic flux density in the magnet: b) Magnetic flux density in the air gap central layer extended to the external area; c) Magnetic flux density in the air gap central line; d) Magnetic flux density in the air gap central layer confined to the magnet.

\section{A. Fringing Effect}

As the magnetic flux alternates between iron and air in the gap region, the flux lines start to become less and less parallel as one moves away from the center of the poles. At the edges of said region the fringing effect starts to be noticeable, leading to a decrease of field uniformity throughout the gap. This happens due to the fact that the permeability of air in the gap region is the same as the permeability of air everywhere else (outside of the core), with their reluctances also being similar. Thus, the flux is shared between the air gap and the neighbouring air volume around it (see Fig. 3). In other words, the flux lines will not be limited to an area Ac corresponding to the section area of the core poles, but rather they will be distributed in a larger fringing area $\mathrm{A}_{\mathrm{f}}$.

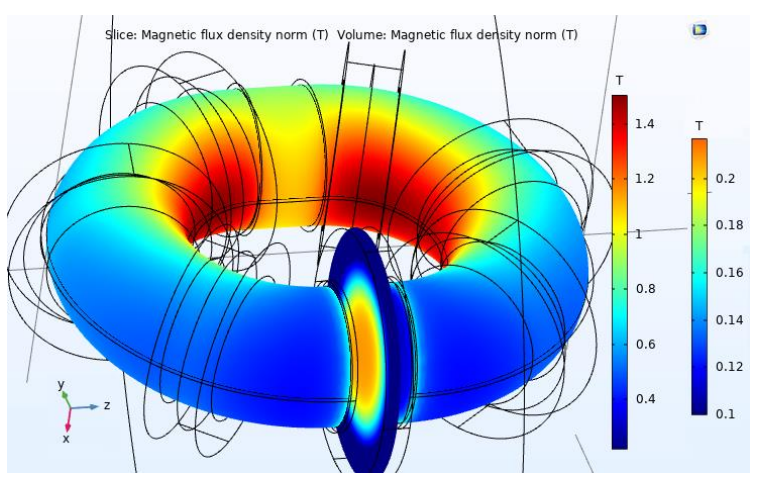

a) Magnetic flux density in the magnet

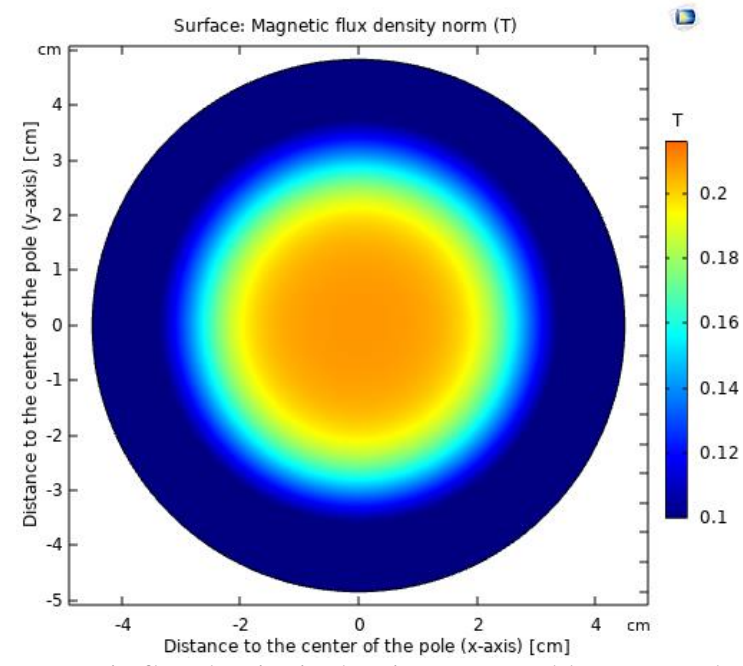

b) Magnetic flux density in the air gap central layer extended to the external area

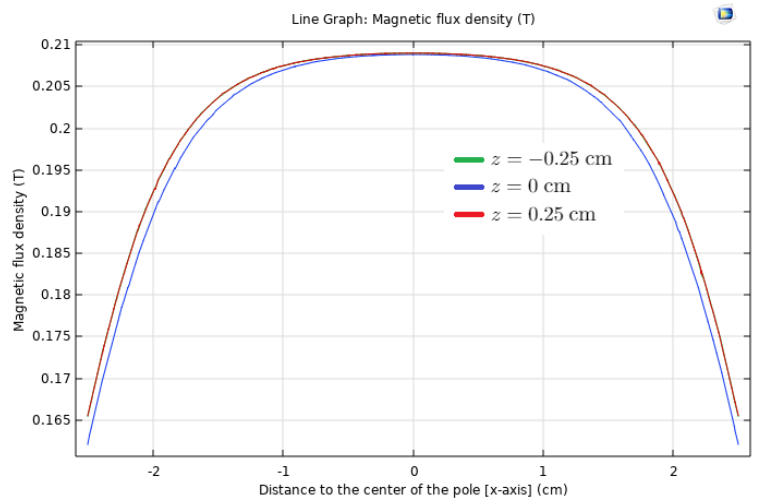

c) Magnetic flux density in the air gap central line

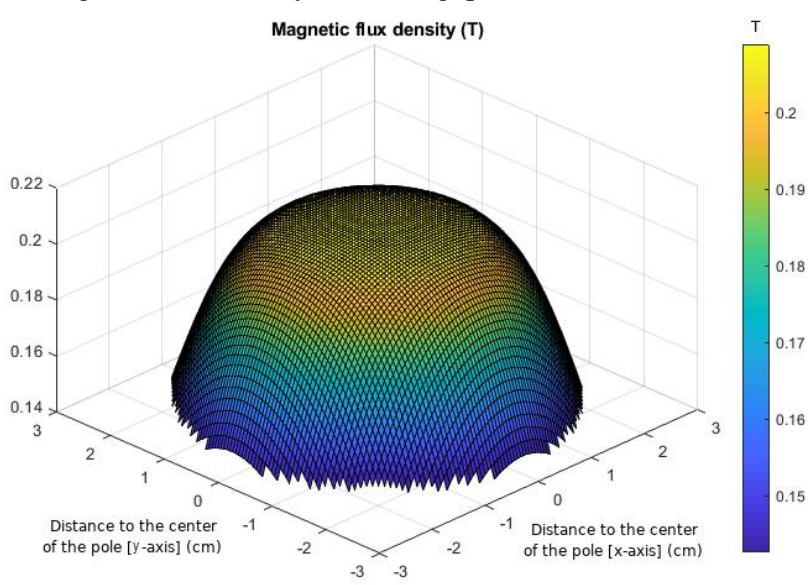

d) Magnetic flux density in the air gap central layer confined to the magnet 


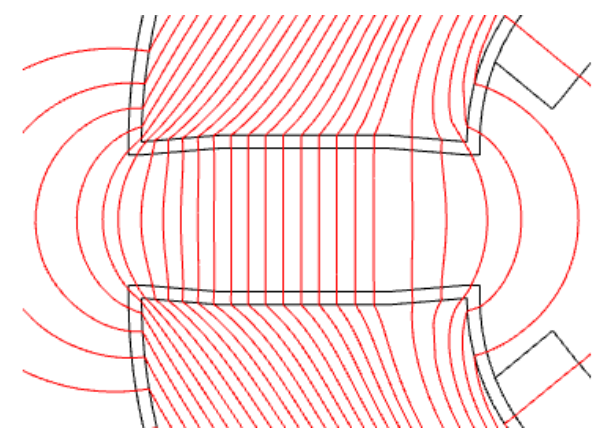

Fig. 3. Detail of the flux lines in the gap region and surrounding air volume, where the fringing effect is noticeable (flux lines in red and magnetic core border in black).

The fringing effect is quantified by a parameter known as the fringing factor, $F_{f}$. Different empirical formulas have been developed to calculate this factor, including one introduced by Roque [14] that follows:

$$
F_{f}=1+\frac{A_{f} B_{f}}{A_{c} B_{g}}
$$

where $B_{g}$ and $B_{f}$ are, respectively, the average magnetic fields in areas $A_{c}$ and $A_{f}$.

\section{B. Cooling}

The electric current used to produce the magnetic field inevitably leads to heating effects in the magnet system, due, to Joule losses in the coils. It is clearly neccessary to properly characterize these effects, in order to minimize any type of negative impact that they may have in the system, such as structural damage due to overpassing the temperatura limuts of the materials of its components, by setting-up an adequate cooling system.

The study of the heating effects was performed only for the magnet system design that presented higher Joule losses, which was the iron fillings magnet core system. By considering this case it was possible to overestimate the cooling requirements, guaranteeing the design of a reliable cooling system.

For this magnet system, the simulated Joule dissipation losses were close to $60 \mathrm{~W}$, for an applied current of $5 \mathrm{~A}$. In fact, this maximum current would only be applied to produce the maximum intensity fields of the fast field cycle (i.e., the polarization and detection fields). However, for simulation purposes, this current was considered constant, which was also a source of overestimation of the cooling requirements. The result of the stationary study performed can be observed in Fig. 4(a), showing the equilibrium temperature of the magnet system without cooling.

As it can be observed that, in the absence of a cooling system, the magnet reaches an average equilibrium temperature of around $390^{\circ} \mathrm{C}$. This high equilibrium temperature makes it clear that it would not be viable to operate the magnet system without a proper cooling system. Otherwise, it would not be possible to manage the dissipated heat, and damage to the components of the magnet would certainly occur.

Taking this into consideration, a cooling system was simulated, by an air flow that removed the excess heat from the magnet system, allowing it to work within a safe temperature range. The defined cooling problem can be summarized as follows: the magnet system was composed of six coils, which dissipated a total of about $60 \mathrm{~W}$; the initial temperature of the system was $20^{\circ} \mathrm{C}$, but the heating effects caused the temperature to rise to around $390^{\circ} \mathrm{C}$; to remove the excess heat, a laminar air flow was forced into the system by a fan, at a flow rate of $0.03 \mathrm{~m}^{3} / \mathrm{s}$ and a temperature of $20^{\circ} \mathrm{C}$; this flow passed through the entire geometry, cooling the system before exiting it via two grilles.

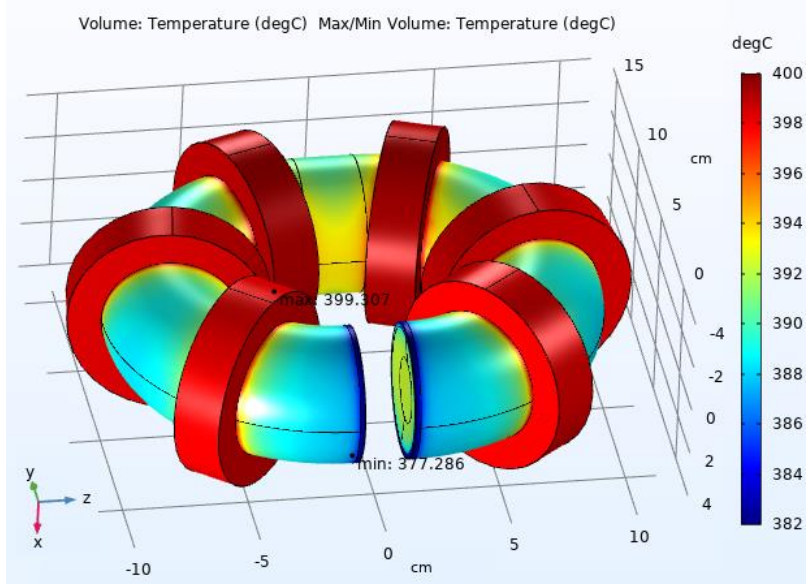

a) Temperature of the magnet without cooling

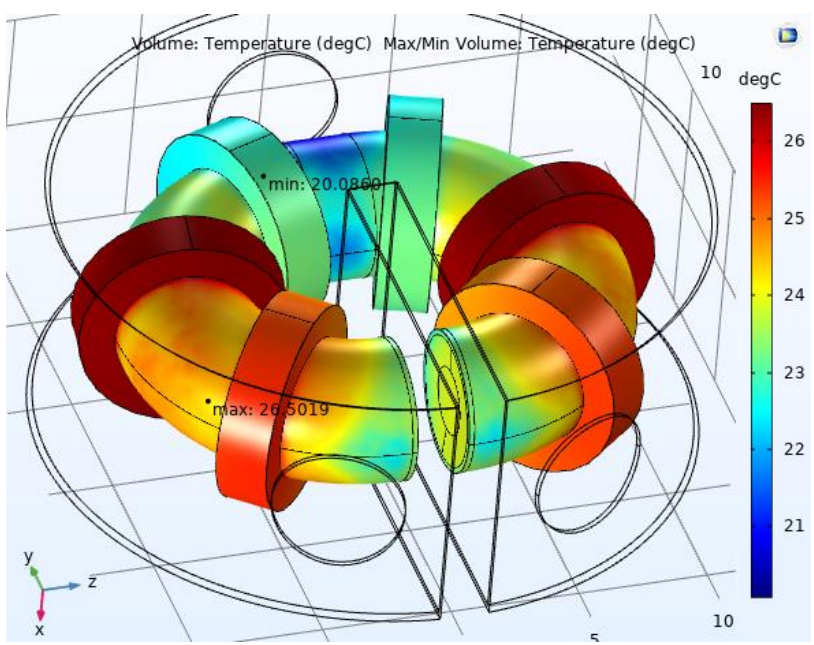

b) Temperature of the magnet with cooling

Fig. 4. Volumetric temperature plot of the magnet core filling system (with customized poles). Equilibrium temperature (a) without cooling and (b) with cooling. In (b), note the casing around the magnet system, where the air flow entry (a fan in the back) and exits (two grilles near the gap) can be seen.

Observing Fig. 4(b), it is possible to note the equilibrium temperature of the system after the cooling effects. Therefore, it is possible, to conclude that the air flow method successful removes the excess heat produced by the coils, as an average equilibrium temperature of around $24{ }^{\circ} \mathrm{C}$ is reached. In conclusion, the magnet system is capable of operating for prolonged periods of time without damage to its component provided that a cooling flow rate of $0.03 \mathrm{~m}^{3} / \mathrm{s}$. Note that different flow rates could have been considered, as there are multiple available fans in the market that can be employed in this system. An increased flow rate would allow to remove more excess heat and, consequently, reduce even further the equilibrium temperature of the system. This would, however, come 
with an increased energy cost, so that this balance needs to be better evaluated when developing the relaxometer.

\section{Magnet Assembly}

To create the prototype of the laminated magnet core system only two components are required: the coils and the thin iron plates (sheets), known as laminations. The laminated core's building (see Fig. 5) was outsourced, being composed of $0.5 \mathrm{~mm}$ thick iron sheets.
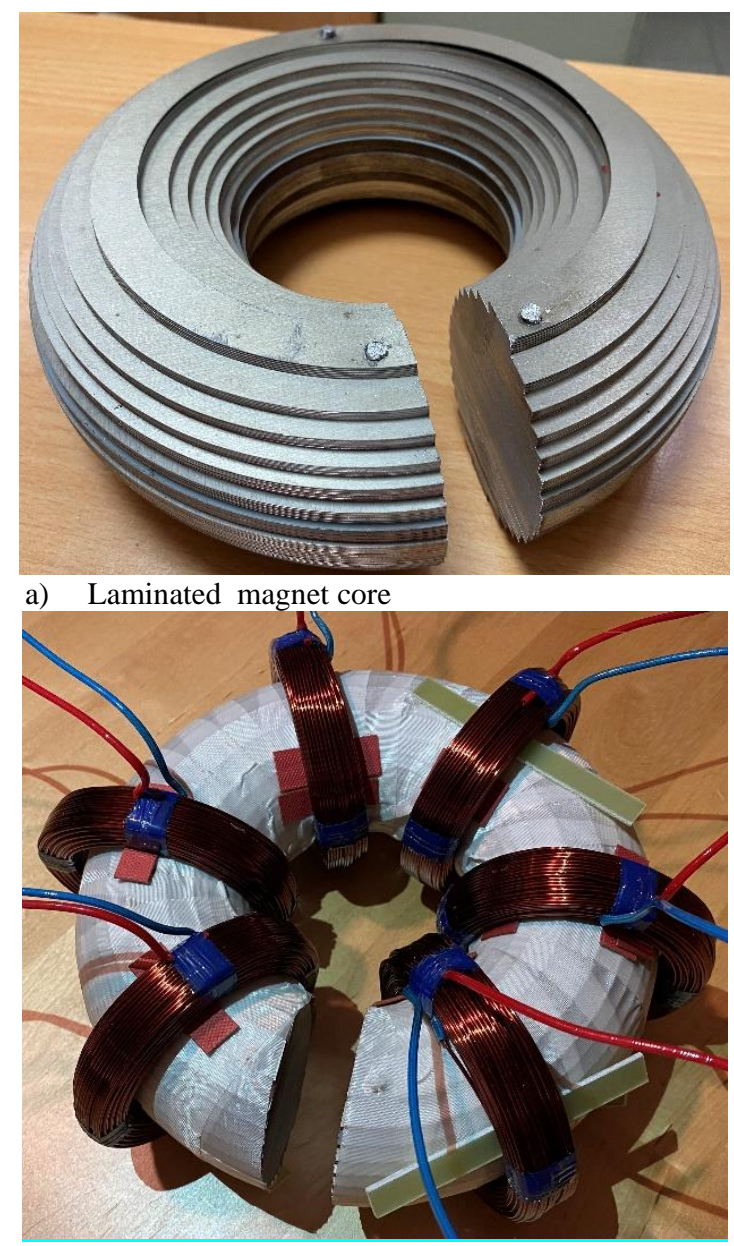

b) Assembled magnet

Fig. 5. Magnet: a) Laminated core; b) Assembled magnet system.

\section{Conclusion}

An innovative FFC NMR magnet system was successfully designed and build with the purpose of enhancing the relaxometer's usability, by increasing its portability capacities. Two solutions in terms of the magnet's core composition were tested and fully characterized in terms of their field homogeneity, fringing effect and heating/cooling effects.

It was possible to disregard the approach of using iron fillings to form the magnet's core since, from a technical standpoint, it was not feasible to develop this magnet with the desired characteristics.

Relatively to other FFC magnets, the main advantages of the designed magnet are: the reduced volume (and weight); the high homogeneity of the induced field in the gap region; the low power demands; and the ability to have complete freedom of rotation in all three axes. The aditional degrees of freedom for samples' insert in the magnet's gap. All these advantages contribute significantly to the increase of the relaxometer's portability capacities and obtain a new concept of FFC NMR relaxometer.

As final reak, it should be pointed out that the proposed magnet layout constitutes a step forward towards the usability of the FFC NMR technique in situ in addition, as by now, in research laboratories.

\section{Acknowledgements}

This work was supported by national funds through Instituto Politécnico de Setúbal, ISEL/Instituto Politécnico de Lisboa, Fundação para a Ciência e a Tecnologia (FCT) with reference UIDB/50021/2020 and UID/CTM/04540/2019, UID/CTM/50025/2019, European Commission project M-ERA-NET2/ 0006/2019 (2017-2020) CELLCOLOR.

\section{References}

[1] A. Abragam, The Principles of Nuclear Magnetism, Oxford University Press, London, (1978).

[2] P. Hore, Nuclear Magnetic Resonance, Oxford University Press, USA, (2015).

[3] F. Noack, "NMR Field-Cycling Spectroscopy: Principles and Applications", Prog. NMR Spectrosc., (1986), 18, pp. 171-276.

[4] R. Kimmich and E. Anoardo, "Field-Cycling NMR relaxometry", Progress in NMR Spectroscopy, (2004), 44, pp. 257-320.

[5] W. Veeman, "Nuclear magnetic resonance, a simple introduction to the principles and applications" Geoderma 80 , 1997, 225- 242.

[6] R. Kimmich, NMR: Tomography, Diffusometry, Relaxometry, Springer Science \& Business Media, (2012).

[7] F. Fujara, D. Kruk, A. Privalov, "Solid State Field-Cycling NMR Relaxometry: Instrumental Improvements and New Applications", Progress in Nuclear Magnetic Resonance Spectroscopy, (2014), 82, 39-69.

[8] E. Anoardo, G. Galli, G. Ferrante, "Fast-Field-Cycling NMR: Applications and Instrumentation", Applied Magnetic Resonance, 2001, 20, (3), pp. 365-404.

[9] M. Levitt, Spin Dynamics: Basics of Nuclear Magnetic Resonance, $2^{\text {nd }}$ edition, Wiley, 2013.

[10] T. C. Farrar, E. D. Becker, Pulse and Fourier transform NMR: Introduction to Theory and Method, Elsevier, (2012).

[11] F. Bloch, Nuclear Induction, Physical review 70, 460-474 (1946).

[12] D. M. Sousa, G. D. Marques, J. Cascais, J. Sebastião, "Desktop Fast-Field Cycling Nuclear Magnetic Resonance Relaxometer", Solid State Nuclear Magnetic Resonance, 2010, 38 (1), pp. 36-43.

[13] P. Videira, "Fast-field cycling nuclear magnetic resonance relaxometer's magnet with optimized homogeneity and reduced volume" (2017), Master Thesis, IST.

[14] A. Roque, "Espectrometro de RMN-CCR com utilização de supercondutores no magneto", (2014), Ph.D. Thesis, IST. 